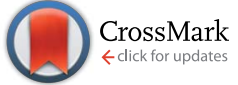

Cite this: RSC Adv., 2016, 6, 43147

\title{
Patterning of amorphous-InGaZnO thin-film transistors by stamping of surface-modified polydimethylsiloxane $\uparrow$
}

\begin{abstract}
Chungwan Gu and Jang-Sik Lee*
An indium gallium zinc oxide (IGZO) layer was patterned and thin-film transistors (TFTs) were fabricated by surface modified polydimethylsiloxane (PDMS) stamping and IGZO solution. The PDMS stamp was prepared by immersion in piranha solution and treatment with UV-ozone to make a hydrophilic surface. Patterned PDMS was inked by contact with the IGZO layer, and then stamped on the desired substrate. The process did not cause etching damage, so the stamped amorphous-IGZO TFTs showed low leakage current of $\sim 10^{-11} \mathrm{~A}$, high on/off current ratio of $\sim 10^{8}$, carrier mobility of $6 \mathrm{~cm}^{2} \mathrm{~V}^{-1} \mathrm{~s}^{-1}$, and narrow hysteresis of $0.2 \mathrm{~V}$. UV irradiation on the IGZO layer caused a photochemical annealing effect that improved the electrical properties of IGZO TFTs. This method provides a simple and versatile process to fabricate transparent metal-oxide TFTs based on patterning the devices by reusable stamping methods.
\end{abstract}

Received 9th March 2016 Accepted 20th April 2016

DOI: $10.1039 / \mathrm{c} 6 \mathrm{ra06264d}$

www.rsc.org/advances

photolithography, ${ }^{11}$ but these methods also have limitations,

\section{Introduction}

Metal-oxide-based thin-film transistors (TFTs), especially amorphous indium gallium zinc oxide (a-IGZO)-based TFTs, have good characteristics including high electron mobility and transparency, and are therefore regarded as essential elements for displays, organic light-emitting diodes (OLEDs) and optoelectronic devices. ${ }^{1-5}$ Recent advances in this field have achieved carrier mobility of higher than $10 \mathrm{~cm}^{2} \mathrm{~V}^{-1} \mathrm{~s}^{-1} \cdot{ }^{6-8}$ Metal-oxidebased TFTs can be fabricated using vacuum processes, such as pulsed laser deposition ${ }^{\mathbf{1}}$ and radio frequency (RF) sputtering. ${ }^{9,10}$ However, vacuum deposition has disadvantages, such as slow fabrication and high cost. As an alternative, solution processes including inkjet printing, ${ }^{11}$ spin-coating, ${ }^{12}$ and dipcoating, ${ }^{13}$ can be used to fabricate devices under ambient conditions.

The patterning process is very important in fabrication and integration of semiconductor devices. Precise fabrication can reduce the leakage current between adjacent devices. Among various patterning technologies, photolithography has been most widely used, but it has disadvantages, such as high production cost and complicated process. In addition, chemicals used to etch thin films can affect the electrical properties, such as carrier mobility, leakage current, threshold voltage, and hysteresis. Printing methods, such as ink-jet printing and electro-hydrodynamic-jet printing, are emerging alternatives to

Department of Materials Science and Engineering, Pohang University of Science and Technology (POSTECH), Pohang 790-784, Republic of Korea. E-mail: jangsik@ postech.ac. $\mathrm{kr}$

$\dagger$ Electronic supplementary information (ESI) available. See DOI: 10.1039/c6ra06264d such as slow fabrication and nozzle clogging.

Recently, patterning techniques on polydimethylsiloxane (PDMS) elastomer such as nano-imprinting and direct transfer of materials have been introduced. Direct transfer of a-IGZO onto PDMS is reported using RF magnetron sputtering. ${ }^{14} \mathrm{ZnO}$ has been patterned by chemical imprinting with a PDMS stamp that had been soaked in ammonia solution and used to selectively dissolve ZnO. ${ }^{15}$ Zinc tin oxide (ZTO) stripe patterns have been fabricated by exploiting capillary force to draw ZTO solution into stripe patterns of PDMS. ${ }^{\mathbf{1 6}}$ However, to fabricate devices these methods still require several steps.

In this research, we suggest PDMS stamping to transfer the IGZO layer for a low-cost and high-speed fabrication method. To give appropriate properties to the PDMS surface, PDMS elastomer was immersed in piranha solution to remove hydrophobic methyl groups. Then the PDMS surface was UV-ozone treated to make it hydrophilic. IGZO solution was transferred by PDMS stamping and subjected to a combination of photochemical and thermal annealing. The IGZO was not damaged by chemical etchant, so patterning decreased leakage current and narrowed the hysteresis loop. The patterned PDMS stamps can be reused many times. We show the possibility of using IGZO solution and PDMS to pattern the active layer of the TFTs.

\section{Experimental}

\section{IGZO solution preparation}

All chemicals were purchased from Sigma-Aldrich and used without further purification. Metal precursors, that is, indium nitrate hydrate $\left(\mathrm{In}\left(\mathrm{NO}_{3}\right)_{3} \cdot x \mathrm{H}_{2} \mathrm{O}, 99.9 \%\right)$, gallium nitrate hydrate $\left(\mathrm{Ga}\left(\mathrm{NO}_{3}\right)_{3} \cdot x \mathrm{H}_{2} \mathrm{O}, \quad 99.9 \%\right)$ and zinc acetate dihydrate 
$\left(\mathrm{Zn}\left(\mathrm{CH}_{3} \mathrm{CO}_{2}\right)_{2} \cdot 2 \mathrm{H}_{2} \mathrm{O}, 98 \%\right)$, were $0.125 \mathrm{M}$ and were mixed at a mole ratio of $\mathrm{In}: \mathrm{Ga}: \mathrm{Zn}=7: 1: 2$. Metal precursors were dissolved in 2-methoxyethanol (2-ME, $\mathrm{CH}_{3} \mathrm{OCH}_{2} \mathrm{CH}_{2} \mathrm{OH}$, anhydrous $99.8 \%$ ) and stirred thoroughly for $6 \mathrm{~h}$ at $75{ }^{\circ} \mathrm{C}$ to make the transparent and homogeneous solution. ${ }^{17}$ Ethylene glycol (EG) was added to synthesize the EG/IGZO solution. The volume ratio was 2 -ME : EG = 20:1. Solution viscosity was controlled by adjusting the amount of EG. IGZO solutions were filtered using $0.2 \mu \mathrm{m}$ syringe filters (PTFE, Whatman) before being spincoated.

\section{Fabrication of patterned PDMS stamps and surface modification}

PDMS elastomer (Sylgard 184) was purchased from Dow Corning. Sylgard 184 base and curing agent in $10: 1$ (w : w) ratio were mixed thoroughly and poured onto the master substrate in a Petri dish. Air bubbles generated during mixing were removed completely in a vacuum chamber. The PDMS was cured at $75^{\circ} \mathrm{C}$ for $6 \mathrm{~h}$, and then peeled off smoothly from the master substrate. ${ }^{\mathbf{1 8 - 2 0}}$ PDMS for IGZO transfer was thermally annealed at $100{ }^{\circ} \mathrm{C}$ for $1 \mathrm{~d}$ in an oven, and then immersed in piranha solution $\left(\mathrm{H}_{2} \mathrm{SO}_{4}: \mathrm{H}_{2} \mathrm{O}_{2}=1: 1 \mathrm{~mol}: \mathrm{mol}\right)$. The PDMS was removed from the solution, followed by a thorough rinse with deionized (DI) water to remove chemical residue. For ozone treatment, the sample was illuminated using a low-pressure mercury lamp (wavelength $\lambda=254 \mathrm{~nm} \mathrm{(90 \% )} \mathrm{and} 185 \mathrm{~nm}$ $(10 \%)$ ) for $30 \mathrm{~min}$.

\section{Fabrication of stamped a-IGZO TFTs}

Highly doped n-type Si wafer with $100 \mathrm{~nm} \mathrm{SiO}_{2}$ was used as the substrate. Before device fabrication the wafers were cleaned in a piranha solution $\left(\mathrm{H}_{2} \mathrm{SO}_{4}: \mathrm{H}_{2} \mathrm{O}_{2}=4: 1 \mathrm{~mol}: \mathrm{mol}\right)$, sonicated sequentially in acetone, ethanol, and DI water for $15 \mathrm{~min}$ each, and then dried under $\mathrm{N}_{2}$ gas. Finally, the wafers were treated with UV-ozone for $10 \mathrm{~min}$ to remove organics and clean the surface.

IGZO solution was spin-coated on the wafer at $500 \mathrm{rpm}$ for 5 $\mathrm{s}$, and then at $3000 \mathrm{rpm}$ for $30 \mathrm{~s}$. The PDMS stamp was attached to a substrate coated with IGZO solution (Fig. 1a). Hydrophilic modified PDMS was put on the IGZO spin-coated layer for several seconds under pressure, and then peeled off and immediately stamped onto the new substrate (Fig. 1b). After peeling off, patterned IGZO on the substrate was annealed photochemically for $1 \mathrm{~h}$, and then annealed thermally at $350{ }^{\circ} \mathrm{C}$ for $1 \mathrm{~h}$ (Fig. 1c). After annealing, Al (100 nm) was deposited through a shadow mask by electron-beam evaporation (Fig. 1d). To compare the effect of PDMS stamping, IGZO TFTs without patterns were also fabricated by spin coating.

\section{Characterization}

The contact angle of IGZO solution on PDMS was measured using the contact angle measurement system (SmartDrop). Images of solution droplets were taken by a high-resolution CCD camera. The electrical properties of TFTs were analyzed in the dark using semiconductor parameter analyzer (KEITHLEY 4200-SCS). Surface roughness and IGZO layer thickness were measured using atomic force microscopy (AFM, VEECO Dimension 3100) and Alpha-Step (KLA-Tencor), respectively.

\section{Results and discussion}

\section{Surface treatment of PDMS}

The PDMS stamp surface is intrinsically hydrophobic with low surface energy because it is coated with nonpolar methyl groups. 2-ME-based IGZO solution is polar so it does not wet well onto PDMS. Dipping PDMS in piranha solution and UVozone treatment made the PDMS surface hydrophilic. Before wet chemical treatment, PDMS was thermally aged at $100{ }^{\circ} \mathrm{C}$ for $1 \mathrm{~d}$ in an oven. Low molecular weight (LMW) species such as uncrosslinked PDMS chains or residual crosslinking chains may be volatilized during thermal aging. ${ }^{21}$ The decrease in the amount of LMW species helps to stabilize the hydrophilic surface after wet chemical treatment. After thermal aging, PDMS surface was first modified by dipping it in piranha solution. Oxygen generated in piranha solution reacted with PDMS to form polar hydrophilic silanol groups. Contact angles decreased with as UV-ozone treatment time increased after dipping the PDMS stamp in piranha solution (Fig. 2). The 185 $\mathrm{nm}$ irradiation induces formation of ozone from oxygen in the air. The organic contamination can also be removed by reaction with ozone driven by irradiation at $\lambda=254 \mathrm{~nm}$. The PDMS surface becomes oxidized and hydrophilized. ${ }^{22,23}$ 2-ME-based IGZO solution was wetted on the PDMS surface. Excessive UVozone treatment (>30 min) caused the PDMS to crack.

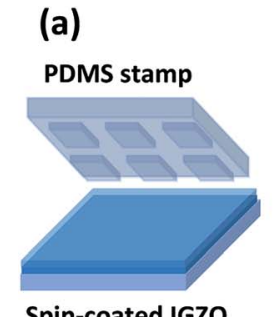

(b)

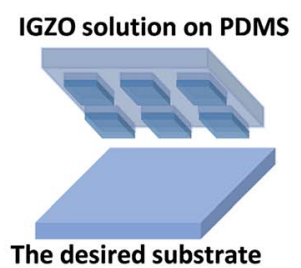

Spin-coated IGZO (c)

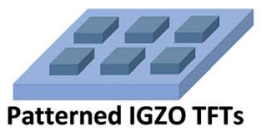

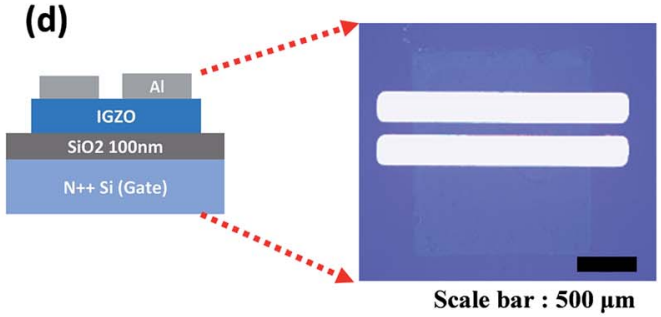

Fig. 1 Schematic of fabrication procedures. (a) PDMS stamp and spin-coated IGZO layer. (b) PDMS stamp with IGZO ink and desired substrate. (c) IGZO transfer to the desired substrate. (d) Cross-sectional view of final device structure. (Right image: optical microscope image of the fabricated device.) 


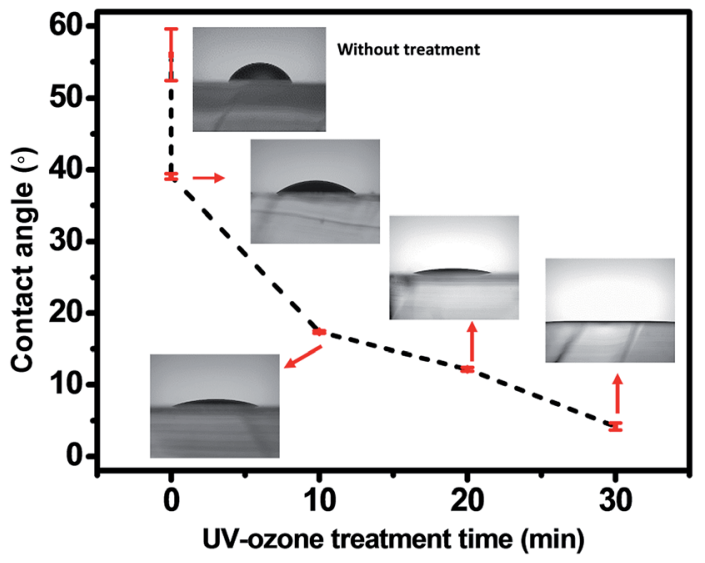

Fig. 2 Contact angles vs. UV-ozone treatment time. Insets: optical images of IGZO solution droplet on PDMS

The volume of solution transferred from PDMS to substrate has an effect on the thickness of active layers. Although the volume of solution transferred cannot be measured precisely, it can be controlled by adjusting stamping pressure. When the PDMS stamp was not surface treated, the transfer failed (Fig. S1†).

\section{Characteristics of stamped IGZO TFTs}

Output and transfer characteristics (Fig. 3) were obtained from the measurement of spin-coated and single-stamped IGZO TFTs. To obtain output curves, drain voltage $\left(V_{\mathrm{d}}\right)$ was swept from $0 \mathrm{~V}$ to $30 \mathrm{~V}$ at fixed gate voltage $\left(V_{\mathrm{g}}\right)$ in increments of $5 \mathrm{~V}$. To obtain transfer curves, $V_{\mathrm{g}}$ was swept from $-20 \mathrm{~V}$ to $30 \mathrm{~V}$ to $-20 \mathrm{~V}$ at $V_{\mathrm{d}}=30 \mathrm{~V}$. Spin-coated IGZO showed threshold voltage $\left(V_{\mathrm{th}}\right)$ of $\sim 3 \mathrm{~V}$ and narrow hysteresis, but high leakage current ( $I_{\text {LEAK }}$ ) (Fig. 3a). In contrast, stamped IGZO showed $I_{\text {LEAK }}$ as low as $10^{-11}$ A with still narrow hysteresis and low $V_{\text {th }}$ (Fig. $3 \mathrm{~b}$ ).

The root mean-square roughness $\left(R_{\mathrm{RMS}}\right)$ of spin-coated and single-stamped IGZO surface was measured using AFM (Fig. 4). The average $R_{\mathrm{RMS}}$ was $0.196 \mathrm{~nm}$ and $0.352 \mathrm{~nm}$ on the spin-
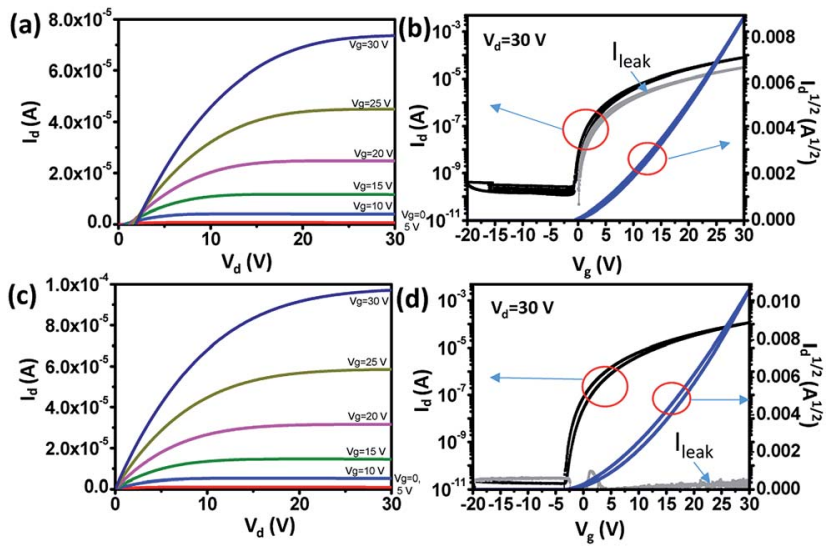

Fig. 3 Electrical properties of IGZO TFTs. (a) Output and (b) transfer characteristics of spin-coated IGZO TFTs. (c) Output and (d) transfer characteristics of stamped IGZO TFTs (single-stamped IGZO).

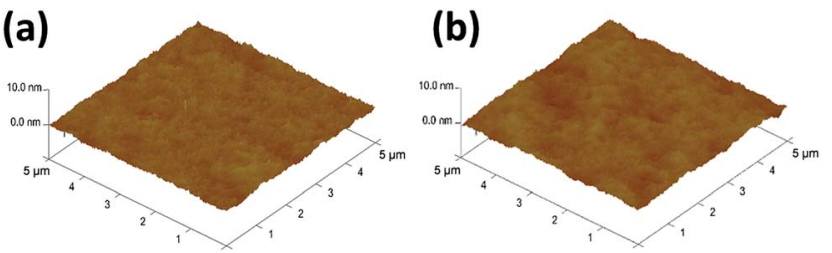

Fig. 4 Atomic force microscopy images of IGZO TFTs. (a) Spin-coated IGZO. (b) Single-stamped IGZO.

coated IGZO surface and stamped IGZO surface, respectively. The thickness of IGZO layer was measured using a stylus profiler. The thickness was $\sim 15 \mathrm{~nm}$ in both the single-stamped and spin-coated layers. The small $R_{\mathrm{RMS}}$ and uniform thickness demonstrate that uniform layers can be synthesized by PDMS stamping. The stamping method transfers IGZO solution directly to the desired substrate and does not require chemicals to etch thin films, whereas conventional device fabrication does. As a result, uniform layers with good electrical properties can be achieved by this stamping method.

To improve electrical properties, photochemical annealing was done. ${ }^{17} \mathrm{UV}$ absorbance of IGZO solution increased near $\lambda=$ $254 \mathrm{~nm}$ (Fig. S2 $\dagger$ ). When UV irradiates IGZO solution, alkoxy groups can be broken by photochemical energies, and metal and oxygen atoms are activated to form metal-oxygen-metal (M-O-M) networks. Compared to thermal annealing alone, photochemical annealing improves the electrical properties (Fig. 5). ${ }^{22,24,25}$

Carrier mobility $\mu$ is calculated using the following equation: ${ }^{26}$
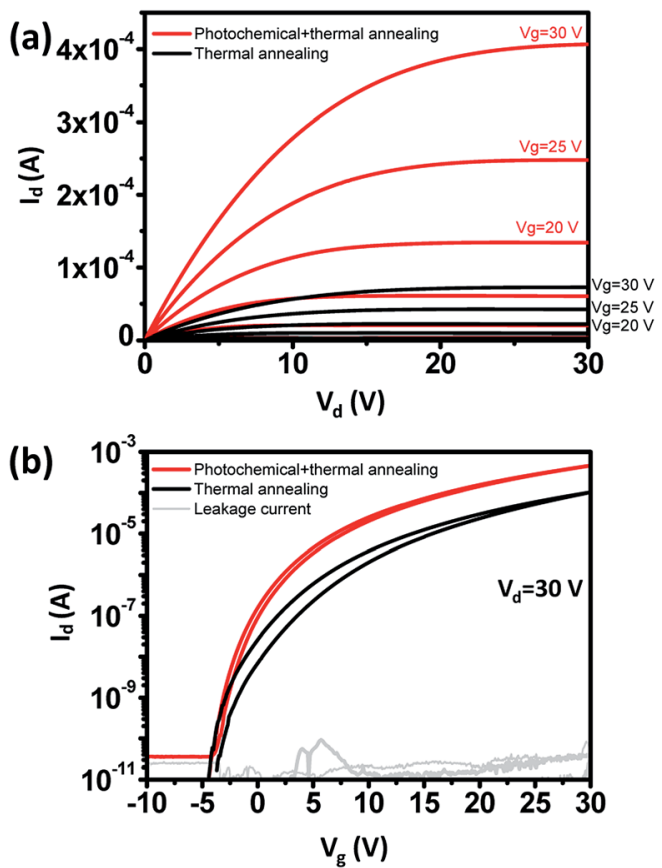

Fig. 5 Electrical properties with and without photochemical annealing of single-stamped IGZO TFTs. (a) Output curves. (b) Transfer curves. Red line, thermal annealing after photochemical annealing. Black line, thermal annealing only. 


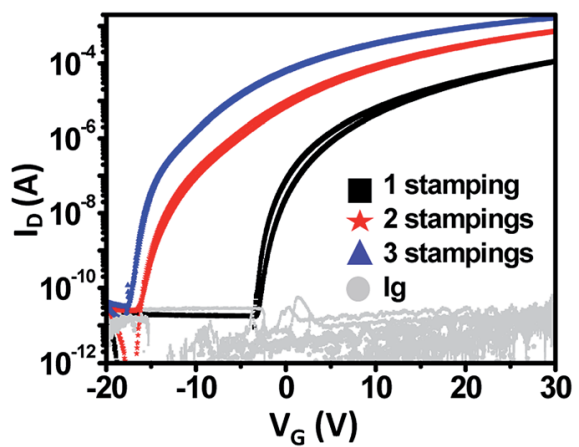

Fig. 6 Electrical characteristics of IGZO TFTs as a function of stamping numbers.

$$
I_{\mathrm{d}}=(W /(2 L)) \mu C_{\mathrm{ox}}\left(V_{\mathrm{g}}-V_{\mathrm{th}}\right)^{2}, V_{\mathrm{d}}>\left(V_{\mathrm{g}}-V_{\mathrm{th}}\right), \text { (saturation) }
$$

where $W$ is channel width, $L$ is channel length, and $C_{\mathrm{ox}}$ is the capacitance of the gate oxide. $V_{\text {th }}$ is calculated from the square root of $I_{\mathrm{d}}$ from the transfer curve. Films that had only been thermally annealed had $\mu=1.6 \mathrm{~cm}^{2} \mathrm{~V}^{-1} \mathrm{~s}^{-1}$, on/off current ratio $=10^{7}$, and $V_{\mathrm{th}}=6.2 \mathrm{~V}$. In contrast, when the IGZO layer had been treated using UV and ozone, thin films can be condensed and densified as a result of the elimination of residual organics. UV-ozone treatment can also decrease the density of defects in IGZO related to oxygen vacancies. ${ }^{25,27}$ As a result, the combination of photochemical and thermal annealing improved the electrical characteristics of IGZO TFTs to $\mu=3.73 \mathrm{~cm}^{2} \mathrm{~V}^{-1} \mathrm{~s}^{-1}$ and $V_{\text {th }} \approx 0.5 \mathrm{~V}$ with narrow hysteresis (Fig. 5).

As the number $n$ of stampings increased, $\mu$ increased and $V_{\text {th }}$ shifted negatively from $6.2 \mathrm{~V}$ to $-6.7 \mathrm{~V}$ (Fig. 6). As $n$ rose, the thickness of IGZO layers increased. When IGZO solution is stamped on the IGZO layer, the pores or pin-holes may be filled by subsequent stampings (Fig. S3†). Accumulation of free carriers and the decreasing number of defects lead to increased $\mu$ and a negative shift in $V_{\mathrm{th}} \cdot{ }^{28}$ These results demonstrate that the electrical properties of the TFT can be controlled by adjusting the number of stacked layers (Fig. S4 $\dagger$ ).

\section{Conclusions}

We developed a-IGZO TFTs by using surface-modified, patterned PDMS to stamp an IGZO layer. The patterned IGZO obtained by PDMS stamping had high $\mu$, low $V_{\text {th }}$ and high on/off ratio. In particular, lower leakage current and hysteresis were obtained with the PDMS stamping method than by conventional spin-coating. PDMS stamping is a simple, inexpensive, and fast fabrication method. In addition, the stamp can be reused. The proposed method may reduce the time and cost of fabricating a-IGZO TFTs, and may also have other applications.

\section{Acknowledgements}

This work was supported by the National Research Foundation of Korea (NRF-2015R1A2A1A15055918), and Future Semiconductor Device Technology Development Program (10045226) funded by the Ministry of Trade, Industry \& Energy
(MOTIE)/Korea Semiconductor Research Consortium (KSRC). In addition, the work was partially supported by the Brain Korea 21 PLUS project (Center for Creative Industrial Materials).

\section{Notes and references}

1 K. Nomura, T. Kamiya, H. Ohta, K. Ueda, M. Hirano and H. Hosono, Appl. Phys. Lett., 2004, 85, 1993.

2 H. Hosono, Thin Solid Films, 2007, 515, 6000-6014.

3 B. D. Ahn, H.-J. Jeon, J. Sheng, J. Park and J.-S. Park, Semicond. Sci. Technol., 2015, 30, 064001.

4 K. Nomura, H. Ohta, A. Takagi, T. Kamiya, M. Hirano and H. Hosono, Nature, 2004, 432, 488-492.

5 J. C. Park, S. Kim, S. Kim, C. Kim, I. Song, Y. Park, U. I. Jung, D. H. Kim and J. S. Lee, Adv. Mater., 2010, 22, 5512-5516.

6 J. H. Na, M. Kitamura and Y. Arakawa, Appl. Phys. Lett., 2008, 93, 063501.

7 J. S. Lee, S. Chang, S. M. Koo and S. Y. Lee, IEEE Electron Device Lett., 2010, 31, 225-227.

8 S. Kim, J. C. Park, D. H. Kim and J. S. Lee, Jpn. J. Appl. Phys., 2013, 52, 041701.

9 J. Yao, N. Xu, S. Deng, J. Chen, J. She, H.-P. D. Shieh, P.-T. Liu and Y.-P. Huang, IEEE Trans. Electron Devices, 2011, 58, 11211126.

10 K. Hoshino, D. Hong, H. Q. Chiang and J. F. Wager, IEEE Trans. Electron Devices, 2009, 56, 1365-1370.

11 S. Jeong, J.-Y. Lee, S. S. Lee, Y.-H. Seo, S.-Y. Kim, J.-U. Park, B.-H. Ryu, W. Yang, J. Moon and Y. Choi, J. Mater. Chem. C, 2013, 1, 4236-4243.

12 Y. S. Rim, H. Chen, Y. Liu, S.-H. Bae, H. J. Kim and Y. Yang, ACS Nano, 2014, 8, 9680-9686.

13 B. N. Pal, P. Trottman, J. Sun and H. E. Katz, Adv. Funct. Mater., 2008, 18, 1832-1839.

14 S. Adachi and S. Okamura, Appl. Phys. Express, 2010, 3, 104101.

15 K. Seong, K. Kim, S. Y. Park and Y. S. Kim, Chem. Commun., 2013, 49, 2783-2785.

16 S. J. Kim, A. Kim, Y. Jo, J.-Y. Yoon, S. S. Lee, Y. Choi, J. Won, S. Nahm, K.-S. Jang and Y. H. Kim, J. Mater. Chem. C, 2014, 2, 8486-8491.

17 Y.-H. Kim, J.-S. Heo, T.-H. Kim, S. Park, M.-H. Yoon, J. Kim, M. S. Oh, G.-R. Yi, Y.-Y. Noh and S. K. Park, Nature, 2012, 489, 128-132.

18 J. K. Hwang, S. Cho, J. M. Dang, E. B. Kwak, K. Song, J. Moon and M. M. Sung, Nat. Nanotechnol., 2010, 5, 742-748.

19 M.-G. Kang and L. J. Guo, J. Vac. Sci. Technol., B: Microelectron. Nanometer Struct., 2008, 26, 2421-2425.

20 M. A. Meitl, Z.-T. Zhu, V. Kumar, K. J. Lee, X. Feng, Y. Y. Huang, I. Adesida, R. G. Nuzzo and J. A. Rogers, Nat. Mater., 2006, 5, 33-38.

21 D. T. Eddington, J. P. Puccinelli and D. J. Beebe, Sens. Actuators, B, 2006, 114, 170-172.

22 Y. Berdichevsky, J. Khandurina, A. Guttman and Y.-H. Lo, Sens. Actuators, B, 2004, 97, 402-408.

23 C. D. Atayde and I. Doi, Phys. Status Solidi C, 2010, 7, 189192. 
24 K. Umeda, T. Miyasako, A. Sugiyama, A. Tanaka, M. Suzuki, E. Tokumitsu and T. Shimoda, J. Appl. Phys., 2013, 113, 184509.

25 B.-Y. Su, A.-H. Cheng, J.-L. Wu, C.-C. Lin, J.-F. Tang, S.-Y. Chu and Y.-D. Juang, J. Disp. Technol., 2015, 11, 6-12.

26 J. S. Lee, S. Chang, S.-M. Koo and S. Y. Lee, IEEE Electron Device Lett., 2010, 31, 225-227.
27 B.-Y. Su, S.-Y. Chu, Y.-D. Juang and H.-C. Chen, Appl. Phys. Lett., 2013, 102, 192101.

28 D. J. Kim, D. L. Kim, Y. S. Rim, C. H. Kim, W. H. Jeong, H. S. Lim and H. J. Kim, ACS Appl. Mater. Interfaces, 2012, 4, 4001-4005. 